\title{
STABILITY WITH A GENERAL RATE FUNCTION FOR A CLASS OF STOCHASTIC EVOLUTION EQUATIONS IN INFINITE DIMENSIONAL SPACES
}

\author{
KAI LIU \\ (Received 12 January 1996; revised 13 September 1996) \\ Communicated by A. G. Pakes
}

\begin{abstract}
The aim of this paper is to investigate the almost sure stability with a certain rate function $\lambda(t)$ for a class of stochastic evolution equations in infinite dimensional spaces under various sufficient conditions. The results obtained here include exponential and polynomial stability as special cases. Much more refined sufficient conditions than the usual ones, for example, those described in [14], are obtained under our framework by the method of Liapunov functions. Two examples are given to illustrate our theory.
\end{abstract}

1991 Mathematics subject classification (Amer. Math. Soc.): primary 93E03; secondary 60H10.

\section{Introduction}

There exists an extensive literature in the stability of stochastic differential equations. We mention here Arnold [1, 2], Carverhill [4], Chappell [5], Chow [6, 7], Crauel [8], Curtain [9, 10], Has'minskii [11] and Mao [15, 16] among others. However, all of the above authors mainly pay attention to exponential stability. Recently, in [13, 14], Mao suggested considering another kind of stability, that is, polynomial stability, which seems to possess great potential for many practical applications. His motivation comes naturally from the following example.

Consider a one-dimensional linear Itô equation

$$
d X_{t}=-\frac{p}{1+t} X_{t} d t+(1+t)^{-p} d W_{t}, \quad t \geq 0
$$

with initial datum $X_{0}=x_{0} \in R$, where $p>1 / 2$ is a constant and $W_{t}$ is one-.

(c) 1997 Australian Mathematical Society 0263-6115/97\$A2.00+0.00 
dimensional Brownian motion. It is easy to obtain the explicit solution

$$
X_{t}=\left(x_{0}+W_{t}\right)(1+t)^{-p}, \quad t \geq 0 .
$$

Therefore for the Liapunov exponent, $\lim \sup _{t \rightarrow \infty} \log \left|X_{t}\right| / t=0$, a.s. However, we have

$$
\limsup _{t \rightarrow \infty} \frac{\log \left|X_{t}\right|}{\log t} \leq-\left(p-\frac{1}{2}\right) \quad \text { a.s. }
$$

In other words, the solution tends to zero almost surely polynomially, although it is not exponentially stable. Therefore a natural question arises: Can we discuss stability with a general rate function instead of exponential or polynomial? Precisely, given a proper positive increasing function $\lambda(t)$, under what assumptions does the solution of a stochastic differential equation tend to zero asymptotically with the given rate function $\lambda(t)$ ?

In this paper, we shall establish almost sure stability with a given rate function $\lambda(t)$ for a class of stochastic differential equations in Hilbert spaces. The results obtained here include exponential and polynomial stability as special cases.

Let us first introduce some necessary notation. Let $H, U, V, K$ be separable Hilbert spaces. Let $\mathscr{L}(U)=\mathscr{L}(U, U)$ be the linear space of all bounded linear operators on $U$, equipped with the usual operator norm. We denote simply by $\|\cdot\|$ the norms in $U, H, \mathscr{L}(U, U)$ when there is no risk of confusion. Let $Q_{M} \in \mathscr{L}(U)$ be a symmetric non-negative operator with $\operatorname{Tr} Q_{M}<+\infty$, the trace of operator $Q_{M}$. It is known that there exists a complete orthonormal basis $\left\{e_{k}\right\}$ in $U$, and a bounded sequence of non-negative real numbers $\lambda_{k} \rightarrow 0$, such that $Q_{M} e_{k}=\lambda_{k} e_{k}, k=1,2, \ldots$

Let $K$ be a real, reflexive, Sobolev type Hilbert space such that the inclusions $K \subset H \subset V=K^{*}$, where $K^{*}$ is the dual of $K$, are densely defined and continuous. Consider the following infinite dimensional stochastic differential equation in $H$ :

$$
d X_{t}=A X_{t} d A_{t}+f\left(X_{t}, t\right) d F_{t}+g\left(X_{t}, t\right) d M_{t}, \quad X_{0}=x_{0} .
$$

Here $F_{t}$ is a $U$-valued, continuous, finite variation process with $F_{0}=0$, and $A_{t}$ is a realvalued non-decreasing, continuous process with $A_{0}=0$. Unless otherwise specified, we will always assume that $M_{t}$ is a $U$-valued, continuous $Q_{M}$-square integrable martingale with $M_{0}=0$, and $A$ is a linear operator, in general unbounded, defined on a dense linear subspace $K:=\mathscr{D}(A)$ of $H$, generating a strong continuous semigroup $S(t)$ of bounded linear operators on $H$. Assume also that $f: H \times R^{+} \rightarrow \mathscr{L}(U, V)$, $g: H \times R^{+} \rightarrow \mathscr{L}(U, H)$ are two continuous, locally bounded mappings. Moreover, we are only concerned with the strong solution $X_{t}$ of equation (1.1); that is, $X_{t}$ is a $K$-valued process. From now on, $\mathscr{L}_{1}(U, H)$ and $\mathscr{L}_{2}(U, H)$ shall denote the spaces consisting of all nuclear operators and Hilbert-Schmidt operators from $U$ into $H$, respectively.

Throughout this paper, we impose the following standing assumption: 
(H1) There exists a symmetric, continuous non-negative $\mathscr{L}_{1}(U, U)$-valued process $Q_{M}(t, \omega)$, a continuous $U$-valued adapted process $Q_{F}(t, \omega)$ and a continuous non-decreasing real-valued process $A_{t}$ with $A_{0}=0$, such that

(1) $\left\langle\left\langle M_{t}\right\rangle\right\rangle=\int_{0}^{t} Q_{M}(s, \omega) d A_{s}$,

(2) $F_{t}=\int_{0}^{t} Q_{F}(s, \omega) d A_{s}$,

where $\left\langle\left\langle M_{t}\right\rangle\right\rangle$ is the tensor quadratic variation of $M_{t}$.

On the other hand, let us introduce the subspace $U_{0}=Q_{M}^{1 / 2} U$ of $U$ which is endowed with the inner product

$$
\langle u, v\rangle_{0}=\sum_{k=1}^{\infty} \frac{1}{\lambda_{k}}\left\langle u, e_{k}\right\rangle_{U}\left\langle v, e_{k}\right\rangle_{U}=\left\langle Q_{M}^{-1 / 2} u, Q_{M}^{-1 / 2} v\right\rangle_{U},
$$

and is also a Hilbert space. An important role will be played by the space of all Hilbert-Schmidt operators $\mathscr{L}_{2}^{0}=\mathscr{L}_{2}\left(U_{0}, H\right)$ from $U_{0}$ into $H$. The space $\mathscr{L}_{2}^{0}$ is also a separable Hilbert space, equipped with the norm

$$
\|\psi\|_{\mathscr{L}_{2}^{0}}^{2}=\operatorname{Tr}\left[\left(\psi \cdot Q_{M}^{1 / 2}\right)\left(\psi \cdot Q_{M}^{1 / 2}\right)^{*}\right]<\infty, \quad \psi \in \mathscr{L}_{2}^{0}
$$

Let $\Phi(t, \omega), t \in[0, T]$, be an $\mathscr{F}_{t}$ measurable $\mathscr{L}_{2}^{0}$-valued process and we define the norm, for arbitrary $t \in[0, T]$,

$$
\begin{aligned}
|\Phi|_{t} & =\left\{E \int_{0}^{t}\|\Phi(s, \omega)\|_{\mathscr{L}_{2}^{0}}^{2} d A_{s}\right\}^{1 / 2} \\
& =\left\{E \int_{0}^{t} \operatorname{Tr}\left(\Phi(s, \omega) \cdot Q_{M}^{1 / 2}\right)\left(\Phi(s, \omega) \cdot Q_{M}^{1 / 2}\right)^{*} d A_{s}\right\}^{1 / 2} .
\end{aligned}
$$

As usual, we also denote the family of all $\mathscr{L}_{2}^{0}$-valued predictable processes $\Phi(t, \omega)$ such that $|\Phi|_{T}<+\infty$ by $N_{w}^{2}\left([0, T] ; \mathscr{L}_{2}^{0}\right)$, or simply by $N_{w}^{2}(0, T)$.

Since Itô's formula in infinite dimensional space plays an important role in the subsequent stability analysis, we shall give a version of it suitable for our purpose without proof (see $[17,18]$ for the details).

THEOREM 1.1 (Itô's formula). Let (, ) and $\langle$,$\rangle denote the inner product in H$ and the duality between $K$ and $V$, respectively. Suppose that $V(x, t)$ is a continuous, locally bounded functional $V: H \times R^{+} \rightarrow R$ admitting derivatives of the first and second-order, denoted by $V_{x}^{\prime}, V_{t}^{\prime}$ and $V_{x x}^{\prime \prime}$, and satisfying:

(1) For each $x \in H, t \in R^{+}, V_{t}^{\prime}(x, t) \in R$ and the derivatives $V_{x}^{\prime}, V_{x x}^{\prime \prime}$ are locally bounded and continuous from $H$ into $H$ and $\mathscr{L}(H, H)$, respectively.

(2) $V_{x}^{\prime}(x, t) \in K, \forall x \in K$ and $\forall y \in V$, the mapping: $x \rightarrow\left\langle V_{x}^{\prime}(x, t), y>i\right.$ is continuous from $K$ into $R$. 
(3) For any $\Gamma \in \mathscr{L}_{1}(H, H)$, the mapping: $x \rightarrow \operatorname{Tr}\left\|V_{x x}^{\prime \prime}(x, t) \Gamma\right\|$, from $K$ into $R$, is continuous.

Moreover, suppose that

(a) $f: H \times R^{+} \rightarrow \mathscr{L}(U, V), \quad g: H \times R^{+} \rightarrow \mathscr{L}_{2}(U, H)$ are two continuous, locally bounded mappings.

(b) $\forall X_{t} \in L^{p}([0, T] \times \Omega, K), p>1, f\left(X_{t}, t\right) \in L^{q}([0, T] \times \Omega, V), 1 / p+1 / q=1$.

(c) $\forall x_{0} \in K,(1.1)$ has a unique strong solution $X_{t}$ such that $g\left(X_{t}, t\right) \in N_{w}^{2}(0, T)$ and $X_{t} \in \mathscr{M}^{p}([0, T] ; K) \cap L^{2}\{\Omega ; C([0, T] ; H)\}$, where $\mathscr{M}^{p}([0, T] ; K)$ denotes the space of all adapted $K$-valued processes $\xi_{t}, t \in[0, T]$ which are jointly measurable in $(t, \omega)$ and are $\mathscr{F}_{t}$-measurable such that $E \int_{0}^{T}\left\|\xi_{t}\right\|^{p} d A_{t}<\infty$, and $C([0, T] ; H)$ denotes the space of $H$-valued continuous functions on $[0, T]$.

Then we have, for arbitrary real-valued non-decreasing process $A_{t}^{\prime}$ with $A_{0}^{\prime}=0$,

$$
\begin{aligned}
V\left(X_{t}, A_{t}^{\prime}\right)=V\left(X_{0}, 0\right) & +\int_{0}^{t} V_{s}^{\prime}\left(X_{s}, A_{s}^{\prime}\right) d A_{s}^{\prime}+\int_{0}^{t}\left(V_{x}^{\prime}\left(X_{s}, A_{s}^{\prime}\right), g\left(X_{s}, s\right) d M_{s}\right) \\
& +\int_{0}^{t}\left\langle V_{x}^{\prime}\left(X_{s}, A_{s}^{\prime}\right),\left(A X_{s}+f\left(X_{s}, s\right) \circ Q_{F}(s)\right) d A_{s}\right\rangle \\
& +\frac{1}{2} \int_{0}^{t} \operatorname{Tr}\left[V_{x x}^{\prime \prime}\left(X_{s}, A_{s}^{\prime}\right)\left(g \circ Q_{M}^{1 / 2}\right)\left(g \circ Q_{M}^{1 / 2}\right)^{*}\right] d A_{s} .
\end{aligned}
$$

In particular, suppose $A_{t}^{\prime}=A_{t}$, and define, for $x \in K$ and $t \in R^{+}$,

$$
\begin{array}{r}
L V(x, t)=V_{t}^{\prime}(x, t)+\left\langle V_{x}^{\prime}(x, t), A x+f(x, t) \circ\left(Q_{F}(t)\right)\right\rangle \\
+\frac{1}{2} \operatorname{Tr}\left[V_{x x}^{\prime \prime}(x, t)\left(g \circ Q_{M}^{1 / 2}\right)\left(g \circ Q_{M}^{1 / 2}\right)^{*}\right] ;
\end{array}
$$

then Itô's formula can also be stated as follows:

$$
V\left(X_{t}, A_{t}\right)=V\left(X_{0}, 0\right)+\int_{0}^{t} L V\left(X_{s}, A_{s}\right) d A_{s}+\int_{0}^{t}\left(V_{x}^{\prime}\left(X_{s}, A_{s}\right), g\left(X_{s}, s\right) d M_{s}\right) .
$$

Next let us give the explicit definition of almost sure stability with rate function $\lambda(t)$.

DEFINITION 1.1. Let $\lambda(\cdot)$ be a real-valued function on $R^{+}$such that $\lambda(t)>0$, $\lambda(t) \uparrow+\infty$ and $\log \lambda(t)$ is uniformly continuous in $t$. Equation (1.1) is said to be almost surely stable with rate function $\lambda(t)$ of order $\delta>0$ if for every $x_{0} \in K$ there exists a finite random variable $\xi(\omega)$ such that

$$
\left\|X_{t}\left(x_{0}\right)\right\| \leq \xi(\omega) \cdot \lambda(t)^{-\delta} \quad \text { a.s. }
$$

for all $t \in R^{+}$, or equivalently,

$$
\limsup _{t \rightarrow \infty} \frac{\log \left\|X_{t}\left(x_{0}\right)\right\|}{\log \lambda(t)} \leq-\delta
$$


In order to prove our main results, we need a Gronwall-type lemma which can be found in [14].

LEMMA 1.1. Let $u(x), n(x)$ belongs to $M\left([0, Y], R^{+}\right)$, the family of all Borel measurable, bounded, left limit non-negative functions defined on $[0, Y], Y=\left(y_{1}, \ldots, y_{n}\right)$ $\in R^{n}$, with $n(x)$ positive and non-decreasing. Let $A_{i}(t)=\left(a_{i 1}(t), \cdots, a_{i n}(t)\right)$, $i=1, \cdots, m$, where $a_{i j}(t):\left[0, y_{i}\right] \rightarrow R^{+}$is a right continuous non-decreasing function with $a_{i j}(0)=0$ for all $i=1, \cdots, m$ and $j=1, \cdots, n$. Let $a\left(x_{1}\right):\left[0, y_{1}\right] \rightarrow R^{+}$ be right continuous and non-decreasing and let $f_{i}(x, s)(i=1,2, \ldots, m)$ be a bounded Borel measurable non-negative function defined on $0 \leq s \leq x \leq Y$ and non-decreasing in $x$. Then the inequality

$$
u(x) \leq a\left(x_{1}\right)+n(x)+\sum_{i=1}^{m} \int_{0}^{x} f_{i}(x, s) u(s-) d A_{i}(s)
$$

implies

$$
u(x) \leq(a(0)+n(x)) \exp \left\{\int_{0}^{x_{1}} \frac{d a\left(\tau_{1}\right)}{a\left(\tau_{1}-\right)+n(x)}+\sum_{i=1}^{m} \int_{0}^{x} f_{i}(x, s) d A_{i}(s)\right\} .
$$

\section{The main results}

In this section we shall use the Liapunov function to study almost sure stability for a class of stochastic differential equations:

$$
d X_{t}=A X_{t} d A_{t}+f\left(X_{t}, t\right) d F_{t}+g\left(X_{t}, t\right) d M_{t}, \quad X_{0}=x_{0} \in K .
$$

Here $f, g, A, F_{t}, M_{t}$ and $V(x, t)$ are defined as in the previous section and are assumed to satisfy the standing hypothesis (H1) and all the conditions in Theorem 1.1. For the purpose of stability analysis, we shall always assume equation (2.1) has a unique strong solution which is denoted by $X_{t}\left(x_{0}\right) \in K$ as in the work of [18].

Since $V(x, t)$ is a $C^{2.1}$-positive function, we define operators $L$ and $Q$ as follows: For $x \in K$ and $t \in R^{+}$, define

$$
\begin{array}{r}
L V(x, t)=V_{t}^{\prime}(x, t)+\left\langle V_{x}^{\prime}(x, t), A x+f(x, t) \circ\left(Q_{F}(t)\right)\right\rangle \\
+\frac{1}{2} \operatorname{Tr}\left[V_{x x}^{\prime \prime}(x, t)\left(g \circ Q_{M}^{1 / 2}\right)\left(g \circ Q_{M}^{1 / 2}\right)^{*}\right]
\end{array}
$$

and

$$
Q V(x, t)=\operatorname{Tr}\left[V_{x}^{\prime} \otimes V_{x}^{\prime}(x, t)\left(g \circ Q_{M}^{1 / 2}\right)\left(g \circ Q_{M}^{1 / 2}\right)^{*}\right] .
$$

At present, we need the following hypothesis in the first two theorems of this section, although we will remove this restriction later on: 
(H2)

$$
X_{t}\left(x_{0}\right) \neq 0 \quad \text { for any } \quad x_{0} \neq 0 \text {. }
$$

Now we can state our main results.

THEOREM 2.1. Let (H2) hold. Suppose $\lambda(t)$ has the property $\lim \sup _{t \rightarrow \infty} \log t / \log \lambda(t)$ $=q \geq 0$. Assume there exists a positive function $V(x, t) \in C^{2.1}\left[(H-\{0\}) \times R^{+}\right] \rightarrow$ $R^{+}$, constants $p>0, u \geq 0, \delta>0, m>0, C \in R$ such that

(1) $\|x\|^{p} \lambda(t)^{m} \leq V(x, t), \quad x \neq 0, \quad t \geq 0$,

(2) $L V(x, t) \leq C V(x, t), \quad x \in K-\{0\}, \quad t \geq 0$,

(3) $\liminf \inf _{t \rightarrow \infty} \log \lambda\left(A_{t}\right) / \log \lambda(t) \geq u, \quad \lim \sup _{t \rightarrow \infty} C A_{t} / \log \lambda(t) \leq m u-\delta-q$, a.s.

Then equation (2.1) is almost surely stable with rate function $\lambda(t)$ of order $\delta / p$, that is,

$$
\limsup _{t \rightarrow \infty} \frac{\log \left\|X_{t}\left(x_{0}\right)\right\|}{\log \lambda(t)} \leq-\frac{\delta}{p} \quad \text { a.s. }
$$

for all $x_{0} \in K-\{0\}$.

PROOF. By Itô's formula and the definitions of $L$ and $Q$, we can show that $\log V\left(X_{t}, A_{t}\right)=\log V\left(x_{0}, 0\right)$

$$
\begin{aligned}
& +\int_{0}^{t} \frac{1}{V\left(X_{s}, A_{s}\right)}\left[L V\left(X_{s}, A_{s}\right)-\frac{1}{2 V\left(X_{s}, A_{s}\right)} Q V\left(X_{s}, A_{s}\right)\right] d A_{s} \\
& +\int_{0}^{t}\left(\frac{1}{V\left(X_{s}, A_{s}\right)} V_{x}^{\prime}\left(X_{s}, A_{s}\right), g\left(X_{s}, s\right) d M_{s}\right) \\
& \leq \log V\left(x_{0}, 0\right)+C A_{t}-\int_{0}^{t} \frac{1}{2 V\left(X_{s}, A_{s}\right)^{2}} Q V\left(X_{s}, A_{s}\right) d A_{s} \\
& +\int_{0}^{t}\left(\frac{1}{V\left(X_{s}, A_{s}\right)} V_{x}^{\prime}\left(X_{s}, A_{s}\right), g\left(X_{s}, s\right) d M_{s}\right) .
\end{aligned}
$$

Due to the exponential martingale inequality [17], we have

$$
\begin{aligned}
P\left\{\omega: \sup _{0 \leq 1 \leq \tau}\right. & {\left[\int_{0}^{t}\left(\frac{1}{V\left(X_{s}, A_{s}\right)} V_{x}^{\prime}\left(X_{s}, A_{s}\right), g\left(X_{s}, s\right) d M_{s}\right)\right.} \\
& \left.\left.-\int_{0}^{t} \frac{v}{2 V\left(X_{s}, A_{s}\right)^{2}} Q\left(X_{s}, A_{s}\right) d A_{s}\right]>\varepsilon\right\} \leq e^{-v \varepsilon}
\end{aligned}
$$

In particular, if

$$
\varepsilon=(1+\sigma) \log k, \quad \nu=1, \quad \tau=k, \quad \sigma>0, \quad(k=1,2, \ldots),
$$


then

$$
\begin{aligned}
P\left\{\omega: \sup _{0 \leq t \leq k}\right. & {\left[\int_{0}^{t}\left(\frac{1}{V\left(X_{s}, A_{s}\right)} V_{x}^{\prime}\left(X_{s}, A_{s}\right), g\left(X_{s}, s\right) d M_{s}\right)\right.} \\
& \left.\left.-\int_{0}^{t} \frac{1}{2 V\left(X_{s}, A_{s}\right)^{2}} Q V\left(X_{s}, A_{s}\right) d A_{s}\right]>(1+\sigma) \log k\right\} \leq \frac{1}{k^{1+\sigma}} .
\end{aligned}
$$

Hence, by the Borel-Cantelli lemma, we deduce that for almost all $\omega \in \Omega$ there exists an integer $k_{0}(\omega)$ such that

$$
\begin{aligned}
& \int_{0}^{t}\left(\frac{1}{V\left(X_{s}, A_{s}\right)} V_{x}^{\prime}\left(X_{s}, A_{s}\right), g\left(X_{s}, s\right) d M_{s}\right) \\
& \quad \leq(1+\sigma) \log k+\int_{0}^{t} \frac{1}{2 V\left(X_{s}, A_{s}\right)^{2}} Q V\left(X_{s}, A_{s}\right) d A_{s}
\end{aligned}
$$

for all $0 \leq t<k, k \geq k_{0}(\omega)$. Substituting this into (2.3) gives

$$
\log V\left(X_{t}, A_{t}\right) \leq \log V\left(x_{0}, 0\right)+C A_{t}+(1+\sigma) \log k
$$

for all $0 \leq t \leq k, k \geq k_{0}(\omega)$ almost surely. Consequently, by hypothesis (1), we have

$$
\begin{aligned}
\frac{\log \left\|X_{t}\right\|}{\log \lambda(t)} & \leq \frac{\log V\left(X_{t}, A_{t}\right)}{p \log \lambda(t)}-\frac{m \log \lambda\left(A_{t}\right)}{p \log \lambda(t)} \\
& \leq \frac{\log V\left(x_{0}, 0\right)}{p \log \lambda(t)}-\frac{1}{p}\left(\frac{m \log \lambda\left(A_{t}\right)}{\log \lambda(t)}-C \frac{A_{t}}{\log \lambda(t)}\right)+\frac{(1+\sigma) \log k}{p \log \lambda(t)} \\
& \leq \frac{\log V\left(x_{0}, 0\right)}{p \log \lambda(t)}-\frac{1}{p}\left(\frac{m \log \lambda\left(A_{t}\right)}{\log \lambda(t)}-C \frac{A_{t}}{\log \lambda(t)}\right)+\frac{(1+\sigma) \log k}{p \log \lambda(k-1)}
\end{aligned}
$$

for all $k-1 \leq t<k, k \geq k_{0}(\omega)$ almost surely.

Letting $\sigma \rightarrow 0$, together with assumption (3), implies

$$
\begin{aligned}
\limsup _{t \rightarrow \infty} \frac{\log \left\|X_{t}\left(x_{0}\right)\right\|}{\log \lambda(t)} & \leq-\frac{1}{p} \liminf _{t \rightarrow \infty}\left(\frac{m \log \lambda\left(A_{t}\right)}{\log \lambda(t)}-C \frac{A_{t}}{\log \lambda(t)}\right)+\frac{q}{p} \\
& \leq-\frac{\delta}{p} \text { a.s. }
\end{aligned}
$$

The proof is complete.

THEOREM 2.2. Let (H2) hold. Suppose $\lambda(t)$ satisfies $\lim \sup _{t \rightarrow \infty} \log t / \log \lambda(t)=$ $q \geq 0$. Assume there exists a positive function $V(x, t) \in C^{2.1}\left[(H-\{0\}) \times R^{+}\right] \rightarrow$ $R^{+}$, constants $p>0, \delta>0, u \geq 0, m>0, C \in R$ and a real-valued predictable process $\xi(t)$ such that 
(1) $\|x\|^{p}(\lambda(t))^{m} \leq V(x, t), \quad x \neq 0, t \geq 0$,

(2) $L V(x, t) \leq C \xi(t) V(x, t), \quad x \in K-\{0\}, t \geq 0$,

(3) $Q V(x, t) \geq \xi(t) V(x, t)^{2}, \quad x \in K-\{0\}, t \geq 0$,

(4) $\liminf _{t \rightarrow \infty} \log \lambda\left(A_{t}\right) / \log \lambda(t) \geq u$, $\lim \sup _{t \rightarrow \infty} \int_{0}^{t} C \xi(s) d A_{s} / \log \lambda(t) \leq m u-\delta-q, \quad$ a.s.

Then equation (2.1) is almost surely stable with rate function $\lambda(t)$ of order $\delta / p$, that is,

$$
\limsup _{t \rightarrow \infty} \frac{\log \left\|X_{t}\left(x_{0}\right)\right\|}{\log \lambda(t)} \leq-\frac{\delta}{p} \quad \text { a.s. }
$$

for all $x_{0} \in K-\{0\}$.

PROOF. Using the same arguments similar to those used in the preceding proofs, it is easy to obtain the following inequality

$\log V\left(X_{t}, A_{t}\right) \leq \log V\left(x_{0}, 0\right)+C \int_{0}^{t} \xi(s) d A_{s}-\int_{0}^{t} \frac{1}{2 V\left(X_{s}, A_{s}\right)^{2}} Q V\left(X_{s}, A_{s}\right) d A_{s}$

$$
+\int_{0}^{\prime}\left(\frac{1}{V\left(X_{s}, A_{s}\right)} V_{x}^{\prime}\left(X_{s}, A_{s}\right), g\left(X_{s}, s\right) d M_{s}\right) \text {. }
$$

Assigning $\varepsilon>0$ arbitrarily and using the exponential martingale inequality, we then have

$$
\begin{aligned}
P\left\{\omega: \sup _{0 \leq t \leq \tau}[\right. & \int_{0}^{t}\left(\frac{1}{V\left(X_{s}, A_{s}\right)} V_{x}^{\prime}\left(X_{s}, A_{s}\right), g\left(X_{s}, s\right) d M_{s}\right) \\
& \left.\left.-\int_{0}^{t} \frac{v}{2 V\left(X_{s}, A_{s}\right)^{2}} Q V\left(X_{s}, A_{s}\right) d A_{s}\right]>r\right\} \leq e^{-v r} .
\end{aligned}
$$

In particular, letting

$$
r=(1+\theta) \varepsilon^{-1} \log k, \quad v=\varepsilon, \quad \tau=k, \quad(k=1,2, \ldots)
$$

where $\theta>0$, we see that there exists an integer $k_{0}(\omega)$, for almost all $\omega \in \Omega$, such that

$$
\begin{aligned}
& \int_{0}^{t}\left(\frac{1}{V\left(X_{s}, A_{s}\right)} V_{x}^{\prime}\left(X_{s}, A_{s}\right), g\left(X_{s}, s\right) d M_{s}\right) \\
& \quad \leq(1+\theta) \varepsilon^{-1} \log k+\int_{0}^{t} \frac{\varepsilon}{2 V\left(X_{s}, A_{s}\right)^{2}} Q V\left(X_{s}, A_{s}\right) d A_{s} .
\end{aligned}
$$

Therefore

$\log V\left(X_{t}, A_{t}\right)$

$$
\leq \log V\left(x_{0}, 0\right)+C \int_{0}^{t} \xi(s) d A_{s}+(1+\theta) \varepsilon^{-1} \log k+\frac{\varepsilon-1}{2} \int_{0}^{t} \frac{Q V\left(X_{s}, A_{s}\right)}{V\left(X_{s}, A_{s}\right)^{2}} d A_{s} .
$$


Letting $\varepsilon=e^{-\delta}, \delta>0$, yields that almost surely

$\log V\left(X_{t}, A_{t}\right)$

$$
\begin{aligned}
& \leq \log V\left(x_{0}, 0\right)+C \int_{0}^{t} \xi(s) d A_{s}+(1+\theta) \varepsilon^{-1} \log k+\frac{\varepsilon-1}{2} \int_{0}^{t} \xi(s) d A_{s} \\
& =\log V\left(x_{0}, 0\right)+e^{\delta}(1+\theta) \log k+C \int_{0}^{t} \xi(s) d A_{s}-\frac{1-e^{-\delta}}{2} \int_{0}^{t} \xi(s) d A_{s}
\end{aligned}
$$

whenever $0 \leq t \leq k, k \geq k_{0}(\omega)$. Therefore it follows from hypothesis (1) that almost surely

$$
\begin{aligned}
& p \log \left\|X_{t}\right\|+m \log \lambda\left(A_{t}\right) \leq \log V\left(X_{t}, A_{t}\right) \\
& \quad \leq \log V\left(x_{0}, 0\right)+e^{\delta}(1+\theta) \log k+C \int_{0}^{t} \xi(s) d A_{s}-\frac{1-e^{-\delta}}{2} \int_{0}^{t} \xi(s) d A_{s} .
\end{aligned}
$$

Hence, letting $\theta \rightarrow 0$ and then $\delta \rightarrow 0$, we can obtain

$$
\begin{aligned}
\frac{\log \left\|X_{t}\left(x_{0}\right)\right\|}{\log \lambda(t)}= & \frac{\log V\left(x_{0}, 0\right)}{p \log \lambda(t)} \\
& -\frac{1}{p \log \lambda(t)}\left[m \log \lambda\left(A_{t}\right)-C \int_{0}^{t} \xi(s) d A_{s}\right]+\frac{\log k}{p \log \lambda(t)} .
\end{aligned}
$$

Now we are in a position to obtain our conclusion:

$$
\begin{aligned}
\limsup _{t \rightarrow \infty} \frac{\log \left\|X_{1}\left(x_{0}\right)\right\|}{\log \lambda(t)} & \leq \frac{1}{p} \limsup _{t \rightarrow \infty} \frac{C \int_{0}^{t} \xi(s) d A_{s}}{\log \lambda(t)}+\frac{q}{p}-\frac{m u}{p} \\
& \leq-\frac{\delta}{p} .
\end{aligned}
$$

The proof is complete.

In what follows, we shall remove hypothesis (H2).

THEOREM 2.3. Suppose $\lambda(t)$ has the property $\lim \sup _{t \rightarrow \infty} \log t / \log \lambda(t)=q \geq 0$. Assume that there exists a positive function $V(x, t) \in C^{2.1}\left[H \times R^{+}\right] \rightarrow R^{+}$, constants $p>0, \delta>0, u \geq 0, m>0, \alpha>0, \beta \geq 0, C \in R$ and a polynomial $\mu(t)$ with positive coefficients such that

(1) $\|x\|^{p} \lambda(t)^{m} \leq V(x, t), \quad x \in H, t \geq 0$,

(2) $L V(x, t) \leq C V(x, t)+\mu(t) e^{C \cdot \alpha t}, \quad x \in K, t \geq 0$,

(3) $Q V(x, t) \leq \mu(t) e^{C \cdot \alpha t} V(x, t), \quad x \in K, t \geq 0$,

(4) $C A_{t} \geq C \alpha t+C \beta, \quad \lim \inf _{t \rightarrow \infty} \log \lambda\left(A_{t}\right) / \log \lambda(t) \geq u$, $\limsup \operatorname{sum}_{t \rightarrow \infty} C A_{t} / \log \lambda(t) \leq m u-\delta-(d+1) q \quad$ a.s., 
where $d$ denotes the degree of the polynomial $\mu(t)$. Then equation (2.1) is almost surely stable with rate function $\lambda(t)$ of order $\delta / p$, that is,

$$
\limsup _{t \rightarrow \infty} \frac{\log \left\|X_{t}\left(x_{0}\right)\right\|}{\log \lambda(t)} \leq-\frac{\delta}{p} \quad \text { a.s. }
$$

for all $x_{0} \in K$.

PROOF. By Itô's formula, we easily have

$$
\begin{aligned}
e^{-C A_{t}} V\left(X_{t}, A_{t}\right)=V\left(x_{0}, 0\right) & +\int_{0}^{t} e^{-C A_{s}}\left[-C V\left(X_{s}, A_{s}\right)+L V\left(X_{s}, A_{s}\right)\right] d A_{s} \\
& +\int_{0}^{t}\left(e^{-C A_{s}} V_{x}^{\prime}\left(X_{s}, A_{s}\right), g\left(X_{s}, s\right) d M_{s}\right) \\
\leq V\left(x_{0}, 0\right) & +\int_{0}^{t} e^{-C A_{s}} \mu(s) e^{C \alpha s} d A_{s} \\
& +\int_{0}^{t}\left(e^{-C A_{s}} V_{x}^{\prime}\left(X_{s}, A_{s}\right), g\left(X_{s}, s\right) d M_{s}\right) .
\end{aligned}
$$

Combined with condition (4), the inequality above implies

$$
\begin{aligned}
e^{-C A_{t}} V\left(X_{t}, A_{t}\right) \leq V\left(x_{0}, 0\right) & +\int_{0}^{t} \mu(s) e^{-C \beta} d A_{s} \\
& +\int_{0}^{t}\left(e^{-C A_{s}} V_{x}^{\prime}\left(X_{s}, A_{s}\right), g\left(X_{s}, s\right) d M_{s}\right) .
\end{aligned}
$$

On the other hand, due to the exponential martingale inequality, we have

$$
\begin{aligned}
P\left\{\omega: \sup _{0 \leq t \leq \tau}\left[\int_{0}^{t}\left(e^{-C A_{s}} V_{x}^{\prime}\left(X_{s}, A_{s}\right), g\left(X_{s}, s\right) d M_{s}\right)\right.\right. \\
\left.\left.-\int_{0}^{t} \frac{v e^{-2 C A_{s}}}{2} Q V\left(X_{s}, A_{s}\right) d A_{s}\right]>r\right\} \leq e^{-v r} .
\end{aligned}
$$

In particular, assigning $\theta>1$ arbitrarily and setting

$$
r=\theta^{(d+1) k+1} \log k, \quad v=\theta^{-(d+1) k}, \quad \tau=\theta^{k}, \quad(k=1,2, \ldots)
$$

where $d$ is the degree of $\mu(t)$, then, by the Borel-Cantelli lemma, we easily deduce that there exists an integer $k_{0}(\omega)$ for almost all $\omega \in \Omega$ such that

$$
\begin{aligned}
& \int_{0}^{t}\left(e^{-C A_{s}} V_{x}^{\prime}\left(X_{s}, A_{s}\right), g\left(X_{s}, s\right) d M_{s}\right) \\
& \quad \leq \theta^{(d+1) k+1} \log k+\frac{\theta^{-(d+1) k}}{2} \int_{0}^{t} e^{-2 C A_{s}} Q V\left(X_{s}, A_{s}\right) d A_{s}
\end{aligned}
$$


for all $0 \leq t \leq \theta^{k}, k \geq k_{0}(\omega)$. Combining this with hypotheses (3) and (4), we have

$$
Q V\left(X_{s}, A_{s}\right) \leq \mu(t) e^{C \alpha t} V\left(X_{s}, A_{s}\right) \leq \mu(t) e^{C A_{t}-\beta C} V\left(X_{s}, A_{s}\right),
$$

which, together with (2.6), implies immediately that almost surely

$$
\begin{aligned}
& \int_{0}^{t}\left(e^{-C A_{s}} V_{x}^{\prime}\left(X_{s}, A_{s}\right), g\left(X_{s}, s\right) d M_{s}\right) \\
& \quad \leq \theta^{(d+1) k+1} \log k+\frac{\theta^{-(d+1) k}}{2} \cdot e^{-C \beta} \int_{0}^{t} \mu(s) e^{-C A_{s}} V\left(X_{s}, A_{s}\right) d A_{s}
\end{aligned}
$$

for all $0 \leq t \leq \theta^{k}, k \geq k_{0}(\omega)$. Substituting this into (2.5) gives

$$
\begin{aligned}
e^{-C A_{t}} V\left(X_{t}, A_{t}\right) & \leq V\left(x_{0}, 0\right)+\theta^{(d+1) k+1} \log k \\
+ & \frac{\theta^{-(d+1) k}}{2} \cdot e^{-C \beta} \int_{0}^{t} \mu(s) e^{-C A_{s}} V\left(X_{s}, A_{s}\right) d A_{s}+e^{-C \beta} \int_{0}^{t} \mu(s) d A_{s}
\end{aligned}
$$

for all $0 \leq t \leq \theta^{k}, k \geq k_{0}(\omega)$. Now we can apply Lemma 1.1 to obtain

$$
\begin{aligned}
& e^{-C A_{t}} V\left(X_{t}, A_{t}\right) \leq V\left(x_{0}, 0\right)+\theta^{(d+1) k+1} \log k \\
& \quad+e^{-C \beta} \mu\left(\theta^{k}\right) A_{\theta^{k}} \cdot \exp \left\{\frac{\theta^{-(d+1) k}}{2} \cdot e^{-C \beta} \mu\left(\theta^{k}\right) A_{\theta^{k}}\right\} \\
& \leq \nu\left(1+\theta^{(d+1) k+1} \log k\right), \quad 0 \leq t \leq \theta^{k}, \quad k \geq k_{0},
\end{aligned}
$$

where $v$ is a positive constant independent of $k$. Consequently,

$$
\frac{e^{-C A_{t}} V\left(X_{t}, A_{t}\right)}{t^{d+1} \log \log t} \leq \frac{v\left(1+\theta^{(d+1) k+1} \log k\right)}{\theta^{(d+1)(k-1)}(\log (k-1)+\log \log \theta)}
$$

for all $\theta^{k-1} \leq t<\theta^{k}, k \geq k_{0}(\omega)$.

This implies

$$
\limsup _{t \rightarrow \infty} \frac{e^{-C A_{t}} V\left(X_{t}, A_{t}\right)}{t^{d+1} \log \log t} \leq v \cdot \theta^{(d+2)}, \quad \text { a.s. }
$$

Letting $\theta$ tends to 1 yields

$$
\limsup _{t \rightarrow \infty} \frac{e^{-C A_{t}} V\left(X_{t}, A_{t}\right)}{t^{d+1} \log \log t} \leq v, \quad \text { a.s. }
$$

Finally,

$$
\begin{aligned}
\frac{\log \left\|X_{t}\right\|}{\log \lambda(t)} & \leq \frac{\log V\left(X_{t}, A_{t}\right)}{p \log \lambda(t)}-\frac{m \log \lambda\left(A_{t}\right)}{p \log \lambda(t)} \\
& \leq \frac{1}{\log \lambda(t)} \cdot \log \left\{e^{C A_{t}} t^{(d+1)} \log \log t \cdot \frac{e^{-C A_{t}} V\left(X_{t}, A_{t}\right)}{t^{d+1} \log \log t}\right\}-\frac{m \log \lambda\left(A_{t}\right)}{p \log \lambda(t)} .
\end{aligned}
$$


Therefore

$$
\begin{aligned}
\limsup _{t \rightarrow \infty} \frac{1}{\log \lambda(t)} \cdot \log \left\|X_{t}\right\| & \leq \frac{1}{p}\left(\limsup _{t \rightarrow \infty} \frac{C A_{t}}{\log \lambda(t)}-m u+(d+1) q\right) \\
& \leq-\frac{\delta}{p} .
\end{aligned}
$$

The proof is complete.

THEOREM 2.4. Suppose that the function $\lambda(t)$ satisfies $\lim \sup _{t \rightarrow \infty} \log t / \log \lambda(t)=$ $q \geq 0$. Assume there exists a positive function $V(x, t) \in C^{2,1}\left[H \times R^{+}\right] \rightarrow R^{+}$, constants $\rho \geq 0, p>0, m>0, u \geq 0, \delta>0, \alpha>0, \beta \geq 0, \mu>0, C \in R$ such that

(1) $\|x\|^{p} \lambda(t)^{m} \leq V(x, t), \quad x \in H, t \geq 0$,

(2) $L V(x, t) \leq C V(x, t)+\mu e^{C \cdot \alpha t+\rho t}, \quad x \in K, t \geq 0$,

(3) $Q V(x, t) \leq \mu e^{C \cdot \alpha t+\rho t} V(x, t), \quad x \in K, t \geq 0$,

(4) $C A_{t} \geq C \alpha t+C \beta, \quad \lim \inf _{t \rightarrow \infty} \log \lambda\left(A_{t}\right) / \log \lambda(t) \geq u, \quad \lim \sup _{t \rightarrow \infty} t / \log \lambda(t)$ $\leq v \leq+\infty, \quad \lim \sup _{t \rightarrow \infty} C A_{t} / \log \lambda(t) \leq m u-\delta-v \rho-q, \quad$ a.s.

Then equation (2.1) is almost surely stable with rate function $\lambda(t)$ of order $\delta / p$, that is,

$$
\limsup _{t \rightarrow \infty} \frac{\log \left\|X_{t}\left(x_{0}\right)\right\|}{\log \lambda(t)} \leq-\frac{\delta}{p} \quad \text { a.s. }
$$

for all $x_{0} \in K$.

PROOF. Using arguments similar to those used in Theorem 2.3, we have

$$
\begin{aligned}
e^{-C A_{t}} V\left(X_{t}, A_{t}\right) \leq V\left(x_{0}, 0\right) & +\int_{0}^{t} \mu e^{-C \beta+\rho s} d A_{s} \\
& +\int_{0}^{t}\left(e^{-C A_{s}} V_{x}^{\prime}\left(X_{s}, A_{s}\right), g\left(X_{s}, s\right) d M_{s}\right) .
\end{aligned}
$$

By the exponential martingale inequality, we can also have

$$
\begin{aligned}
P\left\{\omega: \sup _{0 \leq t \leq \tau}[\right. & \int_{0}^{t}\left(e^{-C A_{s}} V_{x}^{\prime}\left(X_{s}, A_{s}\right), g\left(X_{s}, s\right) d M_{s}\right) \\
& \left.\left.-\int_{0}^{t} \frac{v e^{-2 C A_{s}}}{2} Q V\left(X_{s}, A_{s}\right) d A_{s}\right]>r\right\} \leq e^{-\nu r} .
\end{aligned}
$$

In particular, assigning $\theta>1$ arbitrarily and substituting

$$
r=\theta k e^{\rho k} \log k, \quad \nu=k^{-1} e^{-\rho k}, \quad \tau=k, \quad(k=1,2, \ldots)
$$


into (2.7) and applying the Borel-Cantelli lemma, we deduce that there exists an integer $k_{0}(\omega)$ for almost all $\omega \in \Omega$ such that

$$
\begin{aligned}
& \int_{0}^{t}\left(e^{-C A_{s}} V_{x}^{\prime}\left(X_{s}, A_{s}\right), g\left(X_{s}, s\right) d M_{s}\right) \\
& \quad \leq k \theta e^{\rho k} \log k+\frac{1}{2} k^{-1} e^{-C \beta-\rho k} \int_{0}^{t} e^{\rho s-C A_{s}} V\left(X_{s}, A_{s}\right) d A_{s}
\end{aligned}
$$

for all $0 \leq t \leq k, k \geq k_{0}(\omega)$. Hence we get from (2.7) that almost surely

$$
\begin{aligned}
e^{-C A_{t}} V\left(X_{t}, A_{t}\right) \leq V\left(x_{0}, 0\right) & +k \theta e^{\rho k} \log k+\int_{0}^{t} \mu e^{-C \beta+\rho s} d A_{s} \\
& +\frac{1}{2} k^{-1} e^{-C \beta-\rho k} \int_{0}^{t} e^{\rho s-C A_{s}} V\left(X_{s}, A_{s}\right) d A_{s}
\end{aligned}
$$

This implies

$$
e^{-C A_{t}} V\left(X_{t}, A_{t}\right) \leq v\left(1+k \theta e^{\rho k} \log k\right), \quad 0 \leq t \leq k, k \geq k_{0}
$$

where $v$ is a positive constant independent of $k$. Consequently, whenever $k-1 \leq t \leq$ $k, k \geq k_{0}(\omega)$,

$$
\frac{e^{-C A_{t}} V\left(X_{t}, A_{t}\right)}{e^{\rho t} t \log t} \leq \frac{v\left(1+k \theta e^{\rho k} \log k\right)}{e^{\rho(k-1)}(k-1) \log (k-1)} \quad \text { a.s. }
$$

Therefore

$$
\limsup _{t \rightarrow \infty} \frac{e^{-C A_{t}} V\left(X_{t}, A_{t}\right)}{e^{\rho t} t \log t} \leq v \cdot e^{\rho} \quad \text { a.s. }
$$

and it follows that almost surely

$$
\begin{aligned}
\frac{\log \left\|X_{t}\right\|}{\log \lambda(t)} & \leq \frac{\log V\left(X_{t}, A_{t}\right)}{p \log \lambda(t)}-\frac{m \log \lambda\left(A_{t}\right)}{p \log \lambda(t)} \\
& \leq \frac{1}{\log \lambda(t)} \cdot \log \left(e^{\rho t+C A_{t} t} \log t \cdot \frac{e^{-C A_{t}} V\left(X_{t}, A_{t}\right)}{e^{\rho t} t \log t}\right)-\frac{m \log \lambda\left(A_{t}\right)}{p \log \lambda(t)} .
\end{aligned}
$$

Hence we obtain

$$
\begin{aligned}
\limsup _{t \rightarrow \infty} \frac{1}{\log \lambda(t)} \cdot \log \left\|X_{t}\right\| & \leq \limsup _{t \rightarrow \infty} \frac{1}{p}\left(\frac{C A_{t}}{\log \lambda(t)}+v \rho+q-m u\right) \\
& \leq-\frac{\delta}{p} .
\end{aligned}
$$

The proof is now complete. 
The following theorem is an immediate consequence of the previous two theorems.

THEOREM 2.5. Suppose that the function $\lambda(t)$ satisfies $\lim \sup _{t \rightarrow \infty} \log t / \log \lambda(t)=$ $q \geq 0$. Assume that there exists a positive function $V(x, t) \in C^{2.1}\left[H \times R^{+}\right] \rightarrow R^{+}$, constants $p>0, \rho \geq 0, \lambda>0, u \geq 0, \delta>0, \alpha>0, \beta \geq 0, C \in R$ and a polynomial with positive coefficients $\mu(t)$ such that

(1) $\|x\|^{p} \lambda(t)^{m} \leq V(x, t), \quad x \in H, t \geq 0$,

(2) $L V(x, t) \leq C V(x, t)+\mu(t) e^{C \cdot \alpha t+\rho t}, \quad x \in K, t \geq 0$,

(3) $Q V(x, t) \leq \mu(t) e^{C \cdot \alpha t+\rho t} V(x, t), \quad x \in K, t \geq 0$,

(4) $C A_{t} \geq C \alpha t+C \beta, \quad \liminf \operatorname{in}_{t \rightarrow \infty} \log A_{t} / \log \lambda(t) \geq u, \quad \operatorname{lim\operatorname {sup}_{t\rightarrow \infty }} t / \log \lambda(t)$ $\leq v \leq+\infty, \quad \lim \sup _{t \rightarrow \infty} C A_{t} / \log \lambda(t) \leq m u-\delta-v \rho-q, \quad$ a.s.

Then equation (2.1) is almost surely stable with rate function $\lambda(t)$ of order $\delta / p$, that is,

$$
\limsup _{t \rightarrow \infty} \frac{\log \left\|X_{t}\left(x_{0}\right)\right\|}{\log \lambda(t)} \leq-\frac{\delta}{p} \quad \text { a.s. }
$$

for all $x_{0} \in K$.

PROOF. For any $\varepsilon>0$, there exists a constant $M$ such that $\mu(t) e^{C \alpha t+\rho t} \leq M e^{C \alpha t+\rho t+\varepsilon t}$. Hence the conclusion follows from Theorem 2.4.

\section{Examples}

In this section, we will give two examples to illustrate the results derived in the previous sections.

EXAMPLE 3.1. Consider the following model equation in population biology [18]:

$$
\begin{gathered}
\frac{\partial X(t, x)}{\partial t}=\mu \Delta X+\epsilon\left(\frac{X}{1+|X|}\right) \dot{M}(t, x), \quad t>0, x \in D \subset R^{n} \\
X(0, x)=x_{0},\left.\quad X\right|_{\partial D}=0 .
\end{gathered}
$$

where $\mu, \epsilon$ are positive constants and $D$ is a bounded domain with smooth boundary in $R^{n}$.

Let $H=L^{2}(D), K=H_{0}^{1}(D)$, a Sobolev space with elements satisfying boundary conditions above, $V=K^{*}, f=0, g: u(x) \rightarrow \epsilon(u(x) /(1+|u(x)|))$ from $H$ into $H$, and $M(t, x)$ be an $H$-valued Wiener process with bounded continuous covariance $q(x, y)$. 
Introduce a Liapunov function

$$
V(u, t)=e^{t m} \int_{D} u(x)^{2} d x, \quad m>0 .
$$

It is easy to verify that

$$
\begin{aligned}
& L V(u, t)=m e^{t m} \int_{D} u(x)^{2} d x-2 \mu e^{t m} \int_{D}|\nabla u(x)|^{2} d x \\
&+e^{t m} \int_{D} \epsilon^{2} q(x, x) \frac{u^{2}(x)}{\left(1+|u(x)|^{2}\right.} d x \\
& \leq m e^{t m} \int_{D} u^{2}(x) d x-2 \mu \int_{D}|\nabla u(x)|^{2} d x \cdot e^{t m}+\epsilon^{2}\langle q u, u\rangle e^{t m} .
\end{aligned}
$$

Let $\lambda_{0}=\inf _{u \in H_{0}^{1}}|\nabla u(x)|^{2} /|u(x)|^{2}$ and $q_{0}=\sup _{x \in D}|q(x, x)|$. Thus we obtain

$$
L V(u, t) \leq\left(m-2 \mu \lambda_{0}+\epsilon^{2} q_{0}\right) V(u, t) .
$$

Therefore, if there exists a $\delta>0$ such that $q_{0} \epsilon^{2} \leq 2 \mu \lambda_{0}-\delta$, that is, if $q_{0} \epsilon^{2}<2 \mu \lambda_{0}$, then by Theorem 2.1, equation (3.1) is exponentially stable, that is,

$$
\limsup _{t \rightarrow \infty} \frac{\log \left\|X_{t}\left(x_{0}\right)\right\|}{t} \leq-\frac{\delta}{2} \quad \text { a.s. }
$$

EXAMPLE 3.2. A variation of (3.1) is the following:

$$
\begin{gathered}
\frac{\partial X(t, x)}{\partial t}=v \Delta X+\theta\left(\frac{X}{1+|X|}\right)+p(t) e^{-\mu t}\left(\frac{1}{1+|X|}\right) \dot{M}(t, x), \\
t>0, x \in D \subset R^{n}
\end{gathered}
$$

which satisfies the same initial boundary conditions as before. Here $\nu, \mu, \theta$ are some proper positive constants and $p(t)$ is a polynomial. In contrast with the previous example, $f(u, t)=\theta(u /(1+|u|))$ is non-linear, $g(u, t)=p(t) e^{-\mu t}(1 /(1+|u(x)|))$ and $M(t, x)$ is an $H$-valued Wiener process with continuous covariance $q(x, y)$ such that $\int_{D}|q(x, x)| d x=C<\infty$. Introduce a Liapunov function

$$
V(u, t)=(1+t) \int_{D} u(x)^{2} d x,
$$

and it is easy to verify that

$$
\begin{aligned}
& L V(u, t)=\int_{D} u(x)^{2} d x-2 v(1+t) \int_{D}|\nabla u(x)|^{2} d x \\
&+2 \theta(1+t) \int_{D} \frac{u^{2}(x)}{(1+|u(x)|)} d x \\
&+(1+t) p(t)^{2} e^{-2 \mu t} \int_{D}\left(\frac{1}{1+|u(x)|}\right)^{2} q(x, x) d x \\
& \leq\left(1-2 v \lambda_{0}+2 \theta\right) V(u, t)+4 C(1+t) p^{2}(t) e^{-2 \mu t}
\end{aligned}
$$


and

$$
Q V(u, t) \leq 4 C(1+t) p^{2}(t) e^{-2 \mu t} V(u, t),
$$

where

$$
\lambda_{0}=\inf _{u \in H_{0}^{1}} \frac{|\nabla u(x)|^{2}}{|u(x)|^{2}}
$$

Hence, by Theorem 2.5, we know that if $2 v \lambda_{0}-2 \theta-2 \mu-1>0$, then the equation is almost surely polynomially stable.

\section{Acknowledgement}

The author expresses his thanks to Professor Xuerong Mao for many valuable suggestions.

\section{References}

[1] L. Arnold, Stochastic differential equation: theory and applications (Wiley, New York, 1974).

[2] _ , 'A formula connecting sample and moment stability of linear stochastic systems', SIAM J. Appl. Math. 44 (1984), 793-802.

[3] T. Caraballo, 'Asymptotic exponential stability of stochastic partial differential equations with delay', Stochastics 33 (1990), $27-47$.

[4] A. Carverhill, 'Flows of stochastic dynamical systems: ergodic', Stochastics 14 (1985), 273-317.

[5] M. Chappell, 'Bounds for average Liapunov exponents of gradient stochastic systems', in: Lecture Notes in Math. 1186 (Springer, Berlin, 1984), pp. 292-307.

[6] P. L. Chow, 'Function-space differential equations associated with a stochastic partial differential equation', Indiana Univ. Math. J. 25 (1976), 609-627.

[7] - 'Stability of nonlinear stochastic evolution equations', J. Math. Anal. Appl. 89 (1982), $400-419$.

[8] H. Crauel, 'Liapunov numbers of Markov solutions of linear stochastic systems', Stochastics 14 (1984), 11-28.

[9] R. Curtain (ed), 'Stability of stochastic dynamical systems', in: Lecture Notes in Math. 294 (Springer, Berlin, 1972).

[10] _ 'Stability of stochastic partial differential equation', J. Math. Anal. Appl. 79 (1981), 352369.

[11] R. Z. Has'minskii, 'Necessary and sufficient conditions for the asymptotic stability of linear stochastic systems', Theory Probab. Appl. 12 (1969), 144-147.

[12] U. G. Haussmann, 'Asymptotic stability of the linear Itô equation in infinite dimensional', J. Math. Anal. Appl. 65 (1978), 219-235.

[13] X. Mao, 'Almost sure polynomial stability for a class of stochastic differential equations', Quarterly J. Math. Oxford (2). 43 (1992), 339-348. 
[14] - - Stability of stochastic differential equations with respect to semimartingales, Pitman Res. Notes Math. Ser. 251 (Longman Sci. Tech., Harlow, 1991).

[15] _ Exponential stability of stochastic differential equations (Marcel Dekker, New York, 1994).

[16] - 'Exponential stability for nonlinear stochastic differential equations with respect to semimartingale', Stochastics 28 (1989), 343-355.

[17] M. Métivier, Semimartingales (de Gruyter, Berlin, 1982).

[18] E. Pardoux, Équations aux dérivées partielles stochastiques non linéaires monotones (Thesis, Université Paris XI, 1975).

[19] G. Da Prato and J. Zabczyk, Stochastic equations in infinite dimensions (Cambridge Univ. Press., Cambridge, 1992).

[20] M. Viot, Solutions faibles d'équations aux dérivées partielles stochastique non linéaires (Thesis, Université Paris VI, 1978).

Department of Statistics and Modelling Science

University of Strathclyde

Glasgow G1 1XH, Scotland

U. K.

e-mail address: kai@stams.strath.ac.uk 